\title{
They Tell Me I'm Allergic to Ragweed
}

\author{
Charles S. Barnes Mercedes Amado \\ The Children's Mercy Hospitals and Clinics, and The University of Missouri-Kansas City Medical School, \\ Kansas City, Mo., USA
}

The patient who is skin test positive, or increasingly in current practice, IgE positive to a known and prevalent aeroallergen, but exhibits no symptoms, has always been a conundrum in the practice of allergy. With the increased accessibility to IgE testing, allergists often see persons with positive specific IgE results and are asked to interpret the clinical significance of these tests. In the current era of evidence-based medicine, it is important to have scientific data to support clinical recommendations.

The article by Bodtger et al. [1] in this issue of International Archives of Allergy and Immunology begins to provide some systematic information on this topic. The authors followed 52 skin test-positive subjects (birch and/or grass) who had rhinitis symptoms and 52 similarly skin test-positive subjects who had no rhinitis symptoms. The subjects were followed during 2 successive birch/grass pollen seasons. The authors observed that nearly $10 \%$ of the subjects asymptomatic before the first season became symptomatic before the end of the study. None of a control group of 39 skin test-negative subjects became symptomatic during the study. Also, some of the subjects who had reported previous symptoms lost symptoms during the time of the study. The most comforting thing about these results is that the old, reliable skin test had a good ability to tell that a person will not develop allergic symptoms (negative predictive value 95-100\%). Also, when the skin prick and specific IgE data were examined, those who lost symptoms had smaller skin tests and lower IgE values, and those who acquired symptoms had larger skin tests and higher IgE values. Unfortunately, as most prac- ticing allergists will tell you, the positive predictive value of skin prick testing or IgE levels for determining which of the asymptomatic group will develop symptoms in any subsequent season was not very good (14-27\%).

A few things that the current authors could not control complicated the study. Pollen levels and individual exposures fluctuate from year to year, and symptoms in any one individual are likely to correlate with this varying exposure. As the researchers mentioned, the 2003 birch pollen season was unusually light, but exposure levels should have been adequate. The other variable that is not only uncontrollable but also difficult to quantify or even recognize is individual memory and bias. Eight subjects realized that they had prior symptoms only after the first season started, and 1 subject denied having symptoms despite symptom indications listed in the daily diary. There are probably some very unique and clever methods whereby these and additional uncontrolled variables can be managed by the investigator, but they are either completely impractical or have not been thought of yet. Nonetheless, the current authors have made very creditable efforts toward handling or at least becoming aware of these confounding parameters. The results of this study are bolstered by the time investigators spent to collect symptoms promptly in season and not by longterm recall. Also, the use of 4 methods to evaluate the atopic status including late phase and conjunctiva testing provides additional confidence in the results. A word of praise should also go to the subjects who volunteered for this inconvenient and often uncomfortable testing. Tak-

\section{KARGER}

Fax +4161306 1234

E-Mail karger@karger.ch

www.karger.com (c) 2011 S. Karger AG, Basel

$1018-2438 / 11 / 1553-0189 \$ 38.00 / 0$

Accessible online at:

www.karger.com/iaa
Correspondence to: Dr. Charles S. Barnes

The Children's Mercy Hospitals and Clinics

Kansas City, MO 64108 (USA)

Tel. +1 816235 1975, Fax +1 8163461301

E-Mail cbarnes@cmh.edu 
en all together, these factors give increased creditability to the conclusions of the authors that skin prick testing has excellent negative predictive value and that asymptomatic skin sensitization is a risk factor for allergy development. However, most asymptomatic individuals remain asymptomatic even when no preventive measures are taken.

Although the observations in this article are similar to observations made by practicing allergists on a daily basis, surprisingly, there are relatively few published accounts documenting sensitization and symptom development $[1,2]$. For example, the observation that sensitization to hen's egg is associated with future aeroallergen sensitization and clinical allergy in young children is often made in clinical practice, but there are very few studies, e.g. the German birth cohort data of Kulig et al. [3] that indicates that the persistence of hen's egg sensitization is a good predictor for later sensitization to aeroallergens and allergic airway disease, available to substantiate this observation. The present study provides evidence that sensitization to pollen is predictive of future allergy symptoms. As the article by Bodtger et al. [1] states: 'there is little relevant literature on the predictive capacity of skin testing'. It has been said that 'all physicians conduct research on a daily basis. Most of them die without publishing their findings.' When clinical observations are made, recorded and published in a systematic manner, the practice of allergy makes great strides forward. Unfortunately, the push to see more patients, the trouble involved in obtaining approval from an ethics board and the potential blow to the ego engendered in submitting ones project to vicious peer review all work to forestall dissemination of important knowledge.

Practicing allergists are sometimes asked by patients if most persons in the general population are positive on skin testing. Although it is intuitive, this article clarifies that asymptomatic persons are unlikely to be positive on skin testing or to develop allergies. But, persons who have strongly positive aeroallergen skin tests and/or high IgEspecific tests are at increased risk of developing clinical allergy in the future. As in most of medicine, the history is a very important element for diagnosis and treatment.

References

1 Bodtger U, Assing K, Poulsen LK: A prospective, clinical study on asymptomatic sensitisation and development of allergic rhinitis: high negative predictive value of allergological testing. Int Arch Allergy Immunol 2011;155:289-296.

-2 Schäfer T, Hoelscher B, Adam H, Ring J, Wichmann HE, Heinrich J: Hayfever and predictive value of prick skin test and specific IgE antibodies: a prospective study in children. Pediatr Allergy Immunol 2003;14: 120-129.

3 Kulig M, Bergmann R, Tacke U, Wahn U, Guggenmoos-Holzmann I: Long-lasting sensitization to food during the first two years precedes allergic airway disease. The MAS Study Group, Germany. Pediatr Allergy Immunol 1998;9:61-67. 